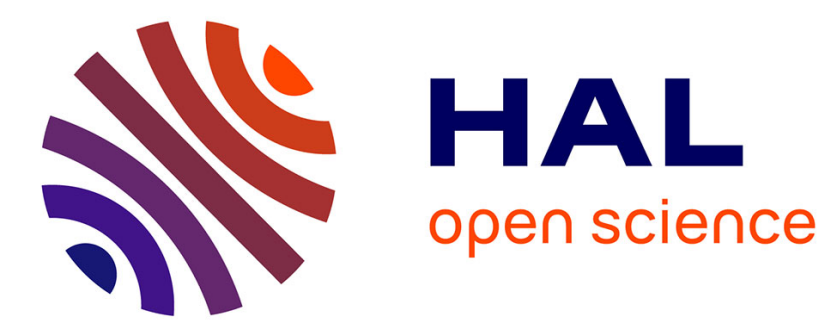

\title{
On the available evidence for the temperature dependence of soil organic carbon
}

\author{
W. Knorr, I. C. Prentice, I. J. House, E. A. Holland
}

\section{To cite this version:}

W. Knorr, I. C. Prentice, I. J. House, E. A. Holland. On the available evidence for the temperature dependence of soil organic carbon. Biogeosciences Discussions, 2005, 2 (4), pp.749-755. hal-00297768

\section{HAL Id: hal-00297768 \\ https://hal.science/hal-00297768}

Submitted on 1 Jul 2005

HAL is a multi-disciplinary open access archive for the deposit and dissemination of scientific research documents, whether they are published or not. The documents may come from teaching and research institutions in France or abroad, or from public or private research centers.
L'archive ouverte pluridisciplinaire HAL, est destinée au dépôt et à la diffusion de documents scientifiques de niveau recherche, publiés ou non, émanant des établissements d'enseignement et de recherche français ou étrangers, des laboratoires publics ou privés. 


\section{On the available evidence for the temperature dependence of soil organic carbon}

W. Knorr ${ }^{1}$, I. C. Prentice ${ }^{1}$, J. I. House ${ }^{1}$, and E. A. Holland ${ }^{2}$

${ }^{1}$ QUEST, Department of Earth Sciences, University of Bristol, Bristol BS8 1RJ, UK

${ }^{2}$ National Center for Atmospheric Research, Boulder, Colorado, USA

Received: 3 June 2005 - Accepted: 22 June 2005 - Published: 1 July 2005

Correspondence to: W. Knorr (wolfgang.knorr@bristol.ac.uk)

(C) 2005 Author(s). This work is licensed under a Creative Commons License.
Temperature

sensitivity of

resistant soil organic matter

W. Knorr et al.

\section{Title Page}

Abstract

Introduction

Conclusions

References

Tables

Figures

14

$\rightarrow$ I

4

Back

Close

Full Screen / Esc

Print Version

Interactive Discussion 


\section{Abstract}

Two recent papers by Knorr et al. (2005) and Fang et al. (2005) provide variations of model fitting conducted in the former study. Knorr et al. (2005) suggested that more recalcitrant fractions of soil organic carbon (SOC) could be more sensitive to temper5 ature. Fang et al. (2005) argue that this is an implication of the choice of model used. Further, Reichstein et al. (2005) point out that the evidence for a stronger temperature sensitivity of recalcitrant soil carbon mainly rests on an analysis of data provided by Kätterer et al. (1998) and argue for a different selection criterion to exclude short-term incubations. Here, we explain why the model used by Knorr et al. (2005) is the simplest

multi-pool model that can fit the available data and is at the same time fully consistent with the concept of "pools", as opposed to some of the model formulations proposed by Fang et al. (2005). It is also pointed out that the criterion proposed by Reichstein et al. (2005) uses posterior information to determine inclusion of experimental data, a practice that should be avoided. We conclude that the original analysis of Knorr et al. (2005) as well as the one added by Fang et al. (2005) indicate that there is a serious possibility that recalcitrant SOC reacts more to temperature changes than labile SOC.

\section{BGD}

2, 749-755, 2005

Temperature

sensitivity of

resistant soil organic matter

W. Knorr et al.

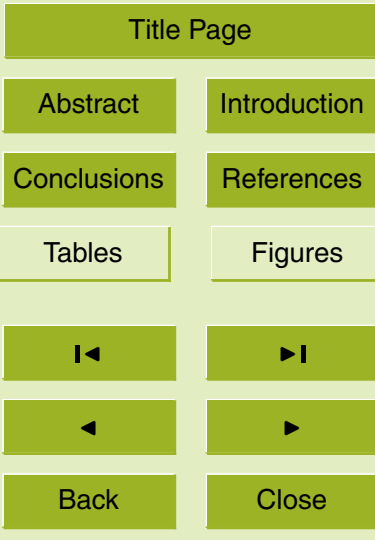

Full Screen / Esc

Print Version

Interactive Discussion 
In their analysis of recent results by Knorr et al. (2005), Fang et al. (2005) provide a number of variations of the model fitting done in the former study. The analysis of Knorr et al. (2005) suggested that more recalcitrant fractions of soil organic carbon (SOC) could be more sensitive to temperature. Fang et al. (2005) argue that this 5 is an implication of the choice of model used, and an alternative analysis does not imply a difference in temperature sensitivity. In a separate comment on the same work, Reichstein et al. (2005) point out that the evidence for a stronger temperature sensitivity of recalcitrant soil carbon mainly rests on an analysis of data provided by Kätterer et al. (1998). They argue that the result would have been different if a different selection 10 criterion had been used to exclude short-term incubations. The question is of major importance for climate research, because most of the terrestrial biosphere's SOC is stored in long-lived, recalcitrant fractions of SOC.

The central message of the work by Knorr et al. (2005) is not questioned, i.e., that a simple model reflecting the extreme heterogeneity of soil carbon can explain all the 15 available evidence from both incubation and warming experiments. No "acclimation", or lack of temperature sensitivity of recalcitrant carbon, is needed to explain the evidence at hand.

In the model of Knorr et al. (2005) each pool decays with a rate given by the Arrhenius equation with two parameters, the activation energy, $E$, and $A$, the reference decay rate

for $E \rightarrow 0$. $A$ is fixed across pools but $E$ is allowed to vary. Fang et al. (2005) show that the fit to the data of Holland et al. (2000) as used by Knorr et al. (2005) and to their own data, is as good if $A$ is allowed to vary across pools, or $E$, or both $A$ and $E$.

This further analysis by Fang et al. (2005) generally supports the conclusions of Knorr et al. (2005). It is especially encouraging to see that the model with constant $A$ across pools can fit both data sets presented as well as a model with both variable $A$ and $E$, even though it has fewer degrees of freedom.

In all of these models, the relative change of the reaction rate, $k$ (the inverse of the turnover time), with respect to temperature, is given by:

$(d k / d T) / k=E / R T^{2}$

BGD

2, 749-755, 2005

Temperature

sensitivity of

resistant soil organic matter

W. Knorr et al.

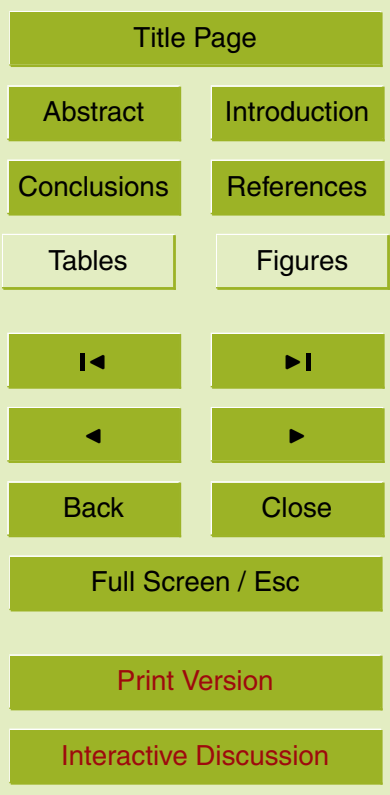

EGU 
and is, at a given temperature, $T$, directly proportional to the activation energy, $E$. (If this value changes by a factor of 1.1 , i.e. increases by $10 \%$, the size of the soil pool in equilibrium will change by the inverse $1 / 1.1=0.91$, i.e. decrease by $9 \%$.) The question is, therefore: do the more recalcitrant SOC fractions have a higher activation energy 5 than the more labile ones?

The central approach followed in Knorr et al. (2005) was to fit the simplest model possible to the data. As Fang et al. (2005) show, adding more degrees of freedom by varying both $A$ and $E$ does not improve the fit. A more principled reason not to consider varying both $A$ and $E$, however, was that such a model would be allowed to become 10 self-contradicting, as it is possible for the different $k(T)$ curves to cross each other, turning an initially more labile into a more stable pool at some cross-over temperature. Even if there are stereochemical reasons for $A$ to differ between soil fractions, as noted by Fang et al. (2005), this argument demonstrates that such differences cannot be represented in a simple pool model without becoming logically inconsistent.

The only other alternative would be to vary $A$, but not $E$.Such a model is less consistent with established notions about reaction kinetics than the reverse: if we vary $E$ and not $A$ we give an implicit explanation for the slower turnover for the pools with higher $E$, while the reverse is not true.

A similar model fit to the original data behind the analysis of Kätterer et al. (1998), where both the rate constants and the temperature sensitivity of two pools are allowed to vary, is presented by Reichstein et al. (2005). This analysis shows a slightly lower $E$ for the labile pool. Here, the same comment applies as for the model fit done by Fang et al. (2005) with both varying $A$ and $E$ : The simplest model that cannot become self-inconsistent is the one used in the analysis of Knorr et al. (2005), with fixed $A$ and varying $E$ across pools. This in itself is not a proof that the slower pools react more strongly to temperature, but it indicates that there is a serious possibility that this might be the case.

To support this possibility, a further analysis was added based on the data presented by Kätterer et al. (1998). Fang et al. (2005) argue that a significant correlation between

\section{BGD}

$2,749-755,2005$

Temperature

sensitivity of

resistant soil organic matter

W. Knorr et al.

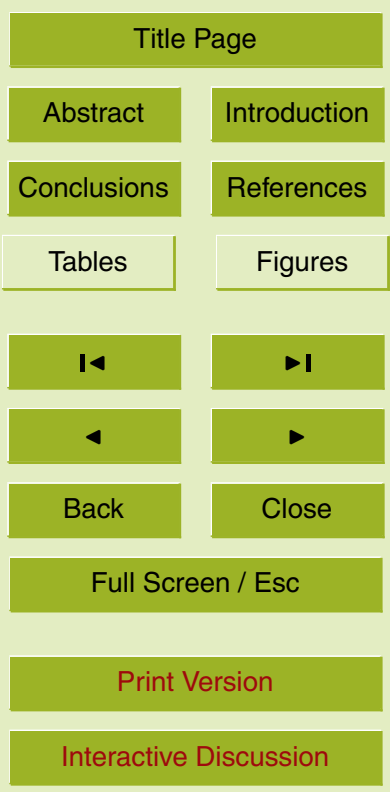

EGU 
the fraction of the fast pool and activation energy based on these data is created by there being two distinct groups, one coming from soil and the other plant material. As Fang et al. (2005) point out, according to Kätterer et al. (1998), plant material has a lower activation energy than SOC. As plant material is a precursor of SOC, i.e. contains 5 more easily degradable components, we believe this supports the stated hypothesis that labile SOC turnover time is less sensitive to temperature change.

Knorr et al. (2005, Fig. 2) also showed the aggregated turnover time at a reference temperature. This value uses not only the relative contribution of the labile and recalcitrant pools, but also the actual values of $A$ and $E$ given by Kätterer et al. (1998). This 10 analysis shows an even steeper dependence of $E$ on turnover time than the model of Knorr et al. (2005). This interpretation, however, is challenged by Reichstein et al. (2005). They demonstrate that a correlation between model parameters in a similar model can be reproduced by adding a constant random "noise" level to synthetic data generated by the model itself. It is evident that, if the model had been linear, such 15 a correlation would not have occurred. We therefore strongly suspect that the result is in itself an artefact of a constant noise level interacting with a model that is highly non-linear. In a realistic experimental setting, however, errors may not be of constant magnitude and vary more strongly with, for example, the measured respiration flux.

A further analysis provided by Reichstein et al. (2005) points out that the positive correlation between activation energy and respiration rate at standard temperature found by Knorr et al. (2005) depended on the criterion which was used to exclude data from incubation experiments that lasted less than, in this case, 100 days. They suggest an alternative selection criterion that relates incubation time to the apparent turnover time of the sample. We believe, however, that a selection criterion that does not only depend on a priori experimental design, but also on a posteriori experimental outcome (in this case the apparent turnover time) contradicts accepted scientific practice. We consequently reject the notion that this be a more appropriate way of analysing the data by Kätterer et al. (1998) suggested by Reichstein et al. (2005). We would, therefore, sustain the conclusion that the data by Kätterer et al. (1998) at least indicate the
BGD

2, 749-755, 2005

Temperature

sensitivity of

resistant soil organic matter

W. Knorr et al.

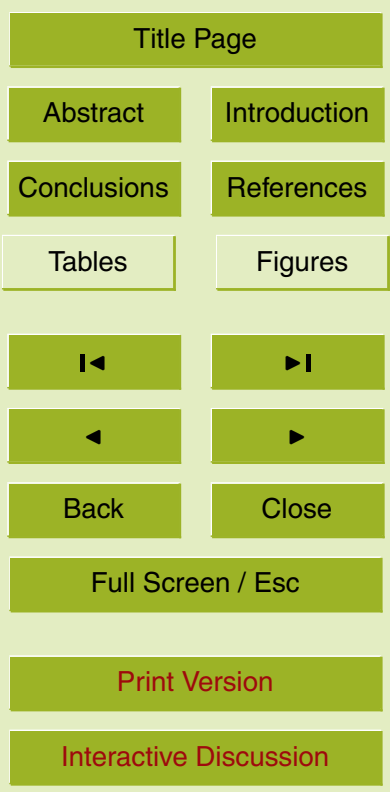

EGU 
possibility of a positive correlation between turnover time and activation energy.

All taken together, we believe that the original analysis of Knorr et al. (2005) as well as the one added by Fang et al. (2005) indicate that there is a serious possibility that recalcitrant SOC reacts more to temperature changes than labile SOC. We agree 5 with Fang et al. (2005) that the turnover time of the complex compounds, of which recalcitrant SOC consists, depends not only on $E$. But it is enough that it does to some degree, to make its decay rate more sensitive to temperature than that of more labile SOC components.

We further disagree with the comment by Reichstein et al. (2005) that the study by 10 Knorr et al. (2005) conveyed an inappropriate level of certainty about the temperature sensitivity of SOC decomposition. In fact, it did not: it simply pointed out that the available evidence is fully consistent with very simple rules of reaction kinetics. Since these very simple rules determine a higher temperature sensitivity of chemically recalcitrant material (Bosatta and Ågren, 1999), it is appropriate to point out the serious possibility

Bosatta, E. and Ågren, G.: Soil organic matter quality interpreted thermodynamically, Soil Biology and Biochemistry, 32, 87-105, 1999.

Fang, C., Smith, P., and Smith, J. U.: Is resistant soil organic matter more sensitive to temperature than the labile organic matter?, Biogeosciences Discuss., 2, 725-735, 2005

Knorr, W., Prentice, I. C., House, J. I, and Holland, E.A.: Long-term sensitivity of soil carbon turnover to warming, Nature, 433, 298-302, 2005.

Kätterer, T., Reichstein, M., Andrén, O., and Lomander, A.: Temperature dependence of organic

BGD

$2,749-755,2005$

Temperature

sensitivity of

resistant soil organic matter

W. Knorr et al.

Title Page

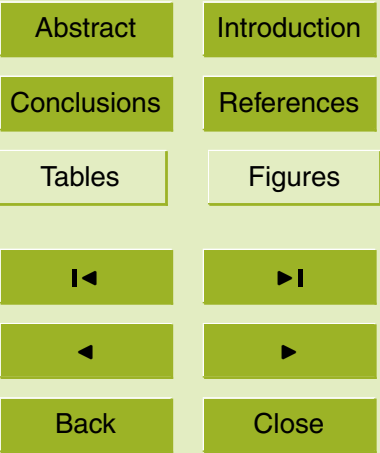

Full Screen / Esc

Print Version

Interactive Discussion 
matter decomposition: a critical review using literature data analyzed with different models, Biol. Fertil. Soils, 27, 258-262, 1998.

Holland, E. A., Neff, J. C., Townsend, A. R., and McKeown, B.: Uncertainties in the temperature sensitivity of decomposition in tropical and subtropical ecosystems: Implications for models, Glob. Biogeochem. Cycl., 14, 1137-1151, 2000.

Reichstein, M., Kätterer, T., Andrén, O., Ciais, P., Schulze, E.-D., Cramer, W., Valentini, R.: Does the temperature sensitivity of decomposition vary with soil carbon pools or quality?, Biogeosciences Discuss., 2, 737-747, 2005

SRef-ID: 1810-6285/bgd/2005-2-737.

BGD

2, 749-755, 2005

Temperature sensitivity of resistant soil organic matter

W. Knorr et al.

Title Page

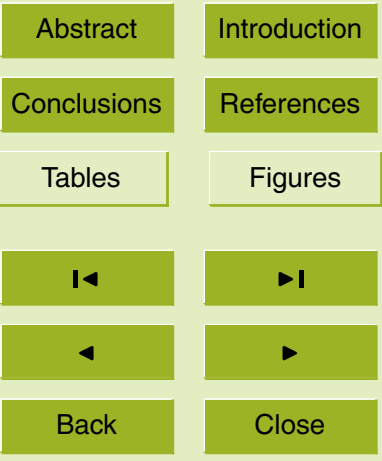

Full Screen / Esc

Print Version

Interactive Discussion 